\title{
Vitessce: a framework for integrative visualization of multi-modal and spatially-resolved single-cell data
}

\author{
Mark S Keller ${ }^{1}$, Ilan Gold ${ }^{1}$, Chuck McCallum ${ }^{1}$, Trevor Manz ${ }^{1}$, \\ Peter V Kharchenko ${ }^{1}$, Nils Gehlenborg ${ }^{1, *}$ \\ ${ }^{1}$ Department of Biomedical Informatics, Harvard Medical School, Boston, MA, USA \\ *Corresponding author: nils@hms.harvard.edu
}

\section{Abstract}

Vitessce is an open-source interactive visualization framework for exploration of multi-modal and spatially-resolved single-cell data, with a modular architecture compatible with transcriptomic, proteomic, genome-mapped, and imaging data types. Its modular, coordinated multiple view implementation facilitates a wide range of visualization tasks to support all common single-cell assays. Vitessce is a client-side web application designed to be integrated with computational analysis tools and data resources and does not require specialized server infrastructure. The software is available at http://vitessce.io.

\section{Main}

Techniques for characterizing tissues and organs at single-cell resolution have recently overcome the challenges of spatial localization and integration of multiple omics assays, improving our ability to distinguish cell types and cell states ${ }^{1}$. The high dimensionality of single-cell data has ushered in a new era of computational models built to automatically assign cells to segmentations, fates, and cell type hierarchies ${ }^{2-4}$. Despite these computational advances, the field of single-cell analysis lacks scalable interactive tools for visual analysis of datasets which integrate genomics, transcriptomics, and imaging modalities. Analyses that bridge modalities, requiring disconnected visualization tools such as standalone image viewers and genome browsers, highlight the need for integrative tools which visually connect diverse biologically derived data types-connections which are key to improving our understanding of human disease dynamics and regulatory mechanisms ${ }^{5}$.

Recent tools for visualization of single-cell data have focused on interactivity and scalability. Tools such as Cirrocumulus ${ }^{6}$, CellXGene ${ }^{7}$, and Pagoda ${ }^{3}$ support interactive exploration of heatmaps and embedding scatter plots with up to millions of cells and thousands of genes. However, these tools are designed for visualization of non-spatial gene expression measurements, lacking support for spatial and genome-mapped modalities, preventing integrative relation of cells across modalities. Further, these and other current web-based single-cell visualization tools require specialized backend servers. Specialized server 
requirements complicate deployments and increase compute infrastructure costs. The lack of sufficient tools for visual analysis of complex and heterogeneous datasets is a problem that has been referred to as the challenge of ultra-scale integrative visualomics ${ }^{8}$. Focusing on individual modalities prevents the achievement of visualization tasks such as comparison and relation for multi-modal datasets, and may limit the utility of current tools in analyses of more complex future assay types.

To overcome these limitations, we developed Vitessce, a scalable web-based single-cell data visualization framework which supports simultaneous visual exploration of integrated transcriptomics, proteomics, genome-mapped, and imaging modalities (see Figure 1). Data from assays such as cellular indexing of transcriptomes and epitopes by sequencing (CITE-seq) ${ }^{9}$, CO-Detection by indEXing (CODEX), multiplexed fluorescence in situ hybridization $(\mathrm{MERFISH})^{10}$, as well as multi-modal assays such as single-nucleus chromatin accessibility and mRNA expression sequencing (SNARE-seq) ${ }^{11}$ can be visualized with Vitessce. We implemented client-side data loaders for accessing files stored on static web servers and cloud object stores, including for Zarr ${ }^{12}$, AnnData ${ }^{4}$, JSON and OME-TIFF ${ }^{13-15}$. Vitessce integrates the previously described $\mathrm{Viv}^{16}$ and HiGlass ${ }^{17}$ toolkits for visualization of multi-scale multiplexed imaging data and genome-mapped data, respectively.

To support visualization of data from a wide range of multi-modal single-cell studies, Vitessce is designed around five key principles:

1. Diverse visual representations. Vitessce supports an extensive collection of interactive visualization components in a configurable grid layout. Through a declarative configuration object, users may define datasets and components of interest, including components tailored for spatially-resolved observations, gene expression matrices, genomic profiles, and two-dimensional embeddings. The spatial visualization component implements a layered visualization approach, supporting multi-scale multiplexed microscopy images, mass spectrometry images, and immunofluorescence images ${ }^{16}$ arranged along with aligned segmentation masks and molecular observations. Genomic profiles, such as those that quantify chromatin accessibility, can be viewed with a genome browser component built upon HiGlass ${ }^{17}$. We have implemented a set of statistical plots which display cell set sizes and gene expression histograms using Vega-Lite ${ }^{18}$. Vitessce is extensible by developers and documentation describing the process of implementing additional visualization or controller components is available.

2. Coordinated multiple views. Visual analysis of single-cell data requires comparison of patterns within and across datasets. The parameters of the multiple views and components available in Vitessce can be coordinated, enabling many-to-many relationships between views and their coordinated parameters. For example, to analyze a SNARE-seq dataset containing chromatin accessibility and mRNA expression information, Vitessce can be configured with genome browser tracks alongside a gene expression heatmap and dimensionality reduction scatterplots. By coordinating all plots to the same selections of cell clusters, Vitessce facilitates visual comparison of clusters 
identified by expression features to those identified by chromatin accessibility features. Another use case aimed at quality control is visual analysis of the performance of three different cell segmentation algorithms. A user may configure a Vitessce instance with three spatial plots coordinated on their zoom levels and center points to synchronize zoom and pan interactions, but independently assign each plot to the segmentations produced by a different algorithm, to facilitate comparison. The implementation of coordinated multiple views in Vitessce builds on a coordination model previously described by Boukhelifa and Rogers ${ }^{19}$.

3. Widgets for Python and R. Exploratory computing environments and computational notebooks such as Jupyter support web-based widget outputs for visualization. We have implemented $\mathrm{R}^{20}$ and Python widgets for Vitessce and object-oriented application programming interfaces (APIs) to simplify the process of writing Vitessce configurations. These widgets can be used with local or remote data, enabling visualization and analysis on a laptop of large datasets that may be located in the cloud or on a compute cluster. The Vitessce Python and R APIs are compatible with the data structures used by popular single-cell data analysis packages, including ScanPy ${ }^{4}$, SnapATAC ${ }^{21}$, and Seurat 2. Cloud and local export functions are included in the Python and R APIs to simplify the data conversion and data transfer steps required for remote data hosting.

4. Component implementation. The modular architecture of Vitessce as one top-level configurable component that wraps individual sub-components for visualization makes reuse and remixing straightforward. We demonstrate that not only can the Vitessce component be bundled into widgets for Python and $R$, but also that the Vitessce component can be integrated into new web apps. The Human Biomolecular Atlas Program (HuBMAP) Portal (http://portal.hubmapconsortium.org) integrates Vitessce for built-in visualization of datasets comprising modalities including single-nucleus ATAC-seq, CODEX, autofluorescence microscopy, and MALDI imaging mass spectrometry ${ }^{22}$. The Vitessce website contains comprehensive documentation to help others develop new tools and web applications around the core set of components.

5. Absence of specialized server-side dependencies. To facilitate integration into a wide range of different software environments (Figure 1), Vitessce was designed to load data stored in static files on web servers rather than relying on a dedicated web application server. Both the web application code and the data files may reside on the same server, simplifying the deployment process. Vitessce can be deployed to environments such as GitHub Pages and scalable object storage systems such as Google Cloud Storage and Amazon Web Services S3.

We demonstrate the importance of these features for visual analysis of multi-modal single-cell experiments through three use cases (Figure 2).

Vitessce enables visual exploration of single-cell experiments which span the modalities of transcriptomics, epigenomics, proteomics, and imaging within a single integrative tool. We 
anticipate that the modular web component architecture of Vitessce will promote scalability, shareability, and accessibility. Future directions include implementing visualization provenance tracking $^{23,24}$ and more robust support for datasets with temporal dimensions. We expect that the community will embrace the extensibility of Vitessce, contributing additional visualization modules and integrating Vitessce into other applications.

\section{Use cases}

\section{Analysis of transcriptomics and imaging data in single cells}

Assays which spatially resolve RNA molecules in a tissue sample provide opportunities to analyze gene expression at subcellular resolution. We demonstrate that Vitessce supports visual analysis of a multiplexed error-robust FISH (MERFISH) dataset alongside corresponding cell segmentations derived by the Baysor method ${ }^{25}$ (Figure 2A). In this experiment, MERFISH identified over 782,000 RNA molecules in a mouse ileum sample. The MERFISH protocol was extended to capture immunofluorescence (IF) images of DAPI stains, facilitating identification of nuclei. In Vitessce, the IF images, cell segmentations, and the spatial positions of transcripts can be explored in a layered spatial visualization. Localization of expression of an individual RNA species can be visualized by selecting and filtering using the included controller components.

Selection of individual genes in Vitessce highlights that many transcripts have distinct spatial distributions throughout the ileum. Through comparison of IF images and transcript positions, we observe that certain transcripts have characteristic intracellular spatial distributions. Neat1 RNAs are enriched in the nucleus, located over the bright DAPI regions in the visualization. A demo of this Vitessce instance is available at https://s3.amazonaws.com/vitessce-data/demos/2021-10-18/8f814e89/index.html?dataset=petu khov-2021-nat-biotechnol.

\section{Exploration of fluorescently-labeled antigens in tissues}

CO-Detection by indEXing (CODEX) is a spatial assay which uses oligonucleotide probes conjugated to antibodies specific to a set of antigens of interest. Through multiple rounds of hybridization, up to 50 different antigens may be imaged. We present an example of using Vitessce to explore a human spleen tissue sample in which 29 antigens were targeted (Figure 2B). The dataset contains the 29-channel microscopy images, cell segmentations, cell-by-antigen quantification, and unsupervised clustering results. To interact with this dataset, we configure Vitessce with a spatial plot, heatmap, and controllers to select image channels, antigens, and cell clusters of interest. With multiple representations of the same data, we can choose to begin exploration by focusing on one of several entity types.

Because this dataset lacks cell type annotations, we are interested in the antigen markers which define each cluster. We approach the visual analysis by selecting a cluster in the cell set 
controller, then searching for cluster-specific patterns in the spatial view. With clustering assignments encoded as cell segmentation mask colors, it appears that cells assigned to cluster 6 within the "Cell K-Means [Mean-All-SubRegions] Expression" localize to a small set of compact regions in the spatial view, suggestive of cellular neighborhoods in the spleen. When we identify cluster 6 in the heatmap view, it is clear that the marker with the highest relative expression in this cluster corresponds to the B cell marker CD20. We can confirm that CD20 is enriched in this spatial region by hiding the cell segmentation masks in the spatial plot, uncovering the underlying image data. Using the spatial layer controller, we can select the CD20 channel and verify that the fluorescence signal indeed appears in the expected regions. The heatmap and image views can help us to find other markers correlated with the CD20 spatial expression pattern. This visualization can be accessed in the HuBMAP Data Portal at https://portal.hubmapconsortium.org/browse/dataset/HBM362.QZWG.832.

The HuBMAP consortium has published CODEX data for several human tissues. Vitessce is embedded in the HuBMAP Data Portal, enabling visual analysis to be performed on any published HuBMAP CODEX dataset, as well as datasets of most other assay types generated by HuBMAP. In the absence of cell type annotations, inferences can be made about unsupervised clustering results. Once HuBMAP dataset processing pipelines assign cell type predictions, enabling verification and relation of cell type annotations across views, Vitessce will be able to display those cell type labels as well.

\section{Comparison of interpolation methods for an imaging mass spectrometry dataset}

Vitessce can be used for visual comparison within datasets containing multiple technical conditions, supporting the workflows of algorithm developers and computational biologists. For example, computational biologists tasked with analyzing imaging mass spectrometry (IMS) data may be interested in the consequences of using different interpolation methods for generation of pyramidal image files. Pyramidal image files contain lower resolution versions of the same image which are generated by pre-aggregating pixels, facilitating multi-scale visualization in a web browser. The choice of a pre-aggregation interpolation function may affect the visual properties of lower resolution images. The open microscopy image processing toolkit bioformats2raw implements several common interpolation methods: GAUSSIAN, AREA, LINEAR, CUBIC, and LANCZOS. We use the multi-scale image rendering capabilities of Vitessce to compare the application of these methods to an IMS dataset (Figure 2C). The zoom levels and centers of each spatial plot are coordinated to facilitate comparison of the same image region at multiple scales. This visualization can be accessed at https://s3.amazonaws.com/vitessce-data/demos/2021-10-18/0beb1473/index.html?dataset=spr aggins-2021-ims. 


\section{Figures}

A

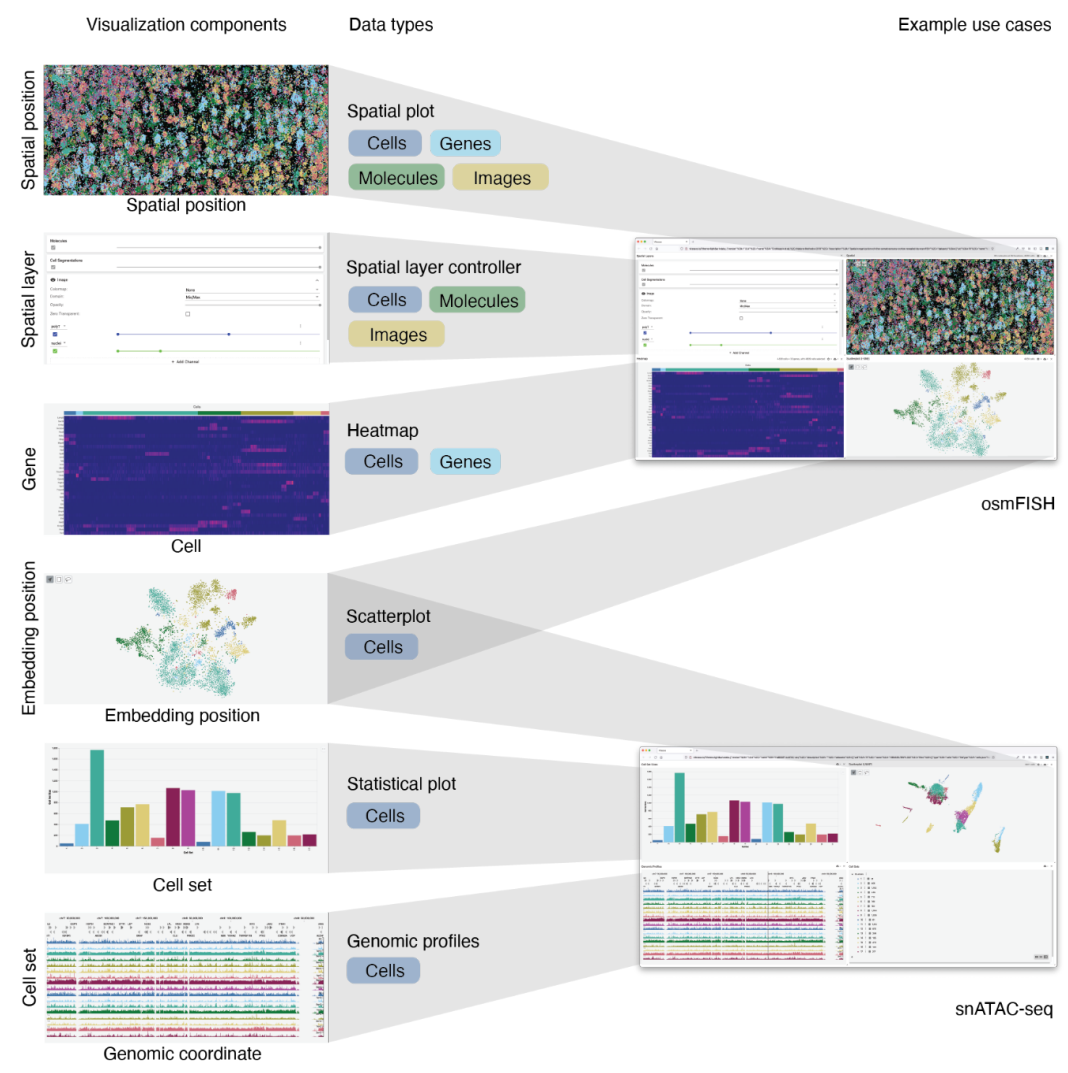

B

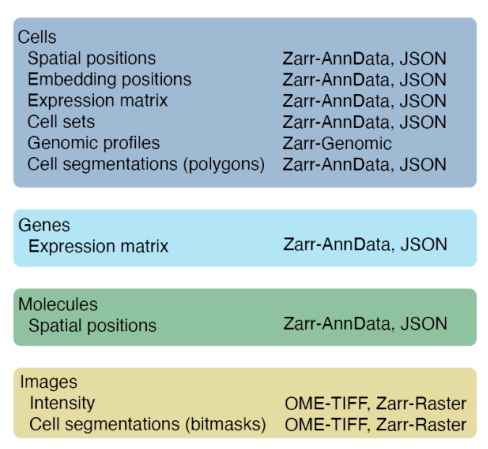

Figure 1. Core visualization components and data types in Vitessce. A) The modular design of Vitessce enables integrative visualization of multi-modal single-cell assays. Left: Single-cell, single-molecule, and microscopy data map onto user-configured visualization components. 
Middle: each visualization component supports visualization of a subset of data types. Right: two example use cases demonstrate that Vitessce can be configured for exploration of a diverse set of single-cell assays. osmFISH: single-molecule fluorescence in situ hybridization ${ }^{26}$; snATAC-seq: single-nucleus assay for transposase-accessible chromatin with high throughput sequencing ${ }^{27}$. B) Vitessce loads datasets containing cells, genes, molecules, and images from multiple file types, including AnnData, JSON, and OME-TIFF.

A

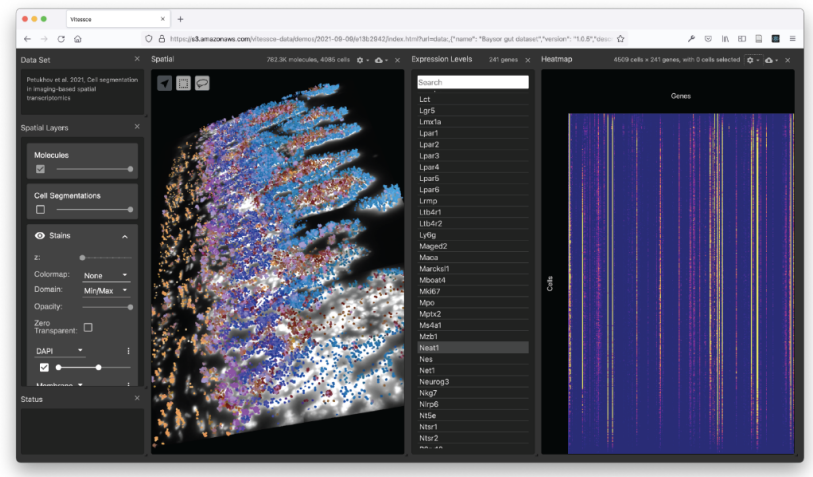

B

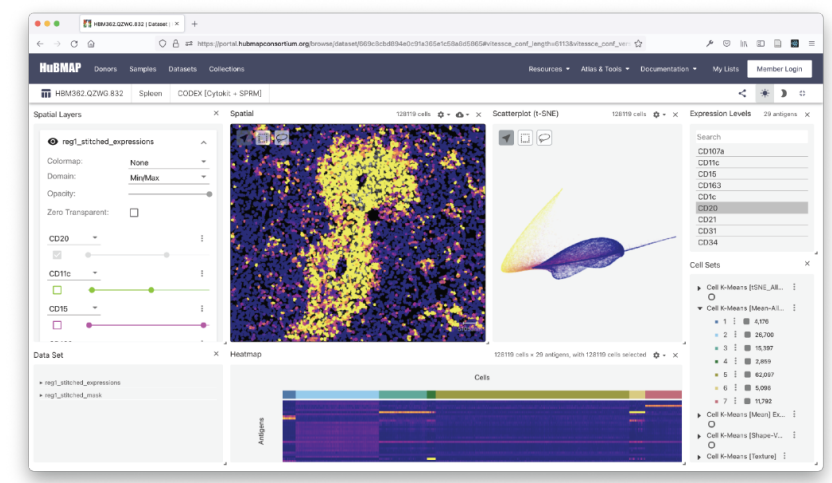

C

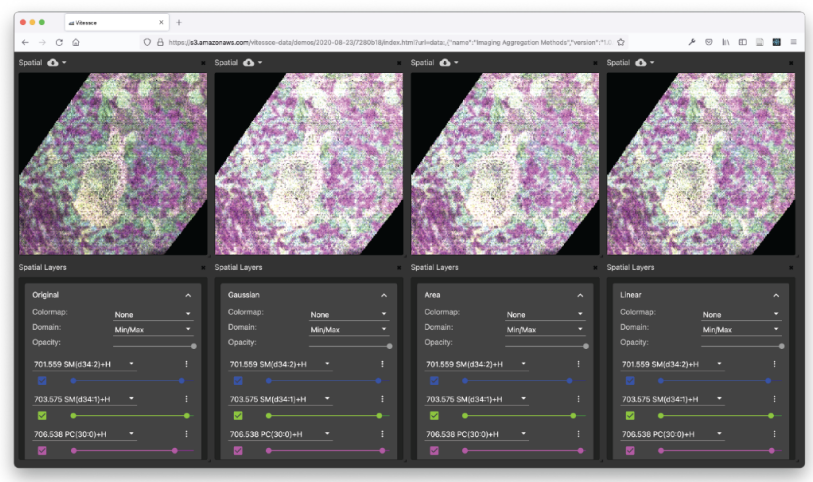

Figure 2. Three use cases of Vitessce. A) A mouse ileum sample ${ }^{25}$ profiled by MERFISH ${ }^{10}$ is visualized with a spatial plot and heatmap. B) A human spleen sample profiled by CODEX is 
visualized with Vitessce embedded in the HuBMAP data portal ${ }^{22}$. C) The outputs of four image interpolation algorithms for an imaging mass spectrometry dataset are visualized in a single Vitessce instance.

\section{Methods}

\section{Processing of example datasets}

\section{Analysis of transcriptomics and imaging data in single cells}

The mouse ileum MERFISH ${ }^{10}$ dataset was obtained from Petukhov et al. ${ }^{25}$ in custom CSV and JSON file formats for transcript spatial positions and cell segmentation polygons, respectively. Petukhov et al. provided staining images in TIFF format. We converted these files into Vitessce-compatible formats (Zarr-AnnData, JSON, OME-TIFF) using Python scripts and the packages Pandas, NumPy ${ }^{28}$, and AnnData ${ }^{4}$. To assign transcripts to cells, we used the Geopandas package to perform a spatial join of the transcript and cell segmentation dataframes with the "intersects" operation.

\section{Exploration of fluorescently-labeled antigens in single cells}

The CODEX dataset was loaded from processed files publicly available on the HuBMAP Data Portal (https://portal.hubmapconsortium.org/browse/dataset/HBM362.QZWG.832). The HuBMAP Portal uses an automated pipeline to process CODEX data which is based on Cytokit $^{29}$ and SPRM (https://github.com/hubmapconsortium/sprm). The pipeline derives cell segmentation bitmasks, two-dimensional embeddings of higher dimensional cell-by-antigen quantification matrices, and cell clusters.

\section{Comparison of interpolation methods for an imaging mass spectrometry dataset}

The IMS dataset was obtained from the HuBMAP Data Portal (https://doi.org/10.35079/hbm876.xnrh.336, MALDI-IMS-pos dataset HBM286.FJBP.622) and processed using the bioformats2raw (https://github.com/glencoesoftware/bioformats2raw) utility. The --downsample-type parameter was used with the values GAUSSIAN, AREA, LINEAR, CUBIC, and LANCZOS to generate an image pyramid for each interpolation algorithm. Each image pyramid was saved as an OME-TIFF file to enable visualization in Vitessce.

\section{Scalable Visualization}

Datasets containing hundreds of thousands of cells and millions of molecules can be rendered efficiently by Vitessce (Supplementary Table 1). The Zarr format for storing chunked and compressed arrays enables Vitessce to load large gene expression matrices and pyramidal images in a web browser. Zarr arrays are stored as directories of static files corresponding to 
compressed array chunks which can be requested and decompressed by a web browser. After decompression, Vitessce uploads chunks of Zarr arrays to the GPU and renders visualizations with DeckGL, Viv (built upon DeckGL), or HiGlass, which are high-level JavaScript frameworks for WebGL-based data visualization. A listing of open-source software packages and web libraries used in Vitessce appears in Supplementary Table 2.

\section{Management of cell sets}

Vitessce considers clusters of cells resulting from unsupervised cluster assignment or manual cell type annotations as cell sets. To facilitate manual cell set annotation within the Vitessce interface, arbitrary sets of cells can be selected and named using the Cell Set Manager component. A lasso selection tool supports selection of any cell set that can be enclosed in a polygon. The set operations of union, intersection, and difference allow additional sets to be derived. Sets can be arranged in hierarchies by clicking and dragging set names in the interface. Annotations can be exported to JSON or CSV formats for interoperability with external tools and algorithms.

\section{Python, $R$, and JavaScript view configuration APIs}

Vitessce configurations can be written using a declarative JSON format or an imperative Python, $\mathrm{R}$, or JavaScript API. Imperative APIs support definition of views, the view layout, and coordinations among views in Python, $\mathrm{R}$, or JavaScript syntax. We anticipate that the native object-oriented paradigms of each language will be more familiar than the lower level JSON format.

\section{Jupyter and $\mathrm{R}$ interactive widgets}

We have packaged the Vitessce JavaScript component as a Python Jupyter notebook widget and an $\mathrm{R}$ htmlwidget. Widgets for Python and $\mathrm{R}$ environments enable Vitessce to be used for visual analysis of local single-cell datasets. The $R$ htmlwidget can be used within $R$ Shiny web applications. The Vitessce widget API assists users in exporting a Vitessce visualization as static files stored on the local machine or a remote web server.

\section{File formats}

Datasets in Vitessce are defined by a set of files loaded over HTTP, each file with its own data type. We have abstracted file reading into loader classes to decouple data types from file types, enabling loading each data type from a range of file types. Data files can be validated against file type schemas, such as JSON schemas. Descriptions of supported file types are provided in Supplementary Table 3. 


\section{Acknowledgments}

Vitessce was developed with funding from the National Institutes of Health (OT2OD026677, T15LM007092, T32HG002295, R33CA263666), the National Science Foundation (DGE1745303), and the Harvard Stem Cell Institute (CF-0014-17-03). The use cases in this manuscript were supported with additional funding from the National Institutes of Health (U54DK120058, 2P41GM103391, OT2OD026671) and National Science Foundation (CBET1828299). We are grateful to Viktor Petukhov for sharing the MERFISH mouse ileum dataset and assisting with its processing and interpretation. We thank Jeffrey Spraggins for the conceptualization of the imaging mass spectrometry technical use case. We would like to thank Sehi L'Yi, John Conroy, Tos Chan, and Jennifer K Marx for feedback and other contributions to the project.

\section{Competing Interests}

N.G. is a co-founder and equity owner of Datavisyn. P.V.K. serves on the Scientific Advisory Board to Celsius Therapeutics, Inc., and Biomage, Inc.

\section{Code availability}

Vitessce is open source and available under an MIT license. We provide the Vitessce JavaScript library at https://github.com/vitessce/vitessce. Vitessce is available as a JavaScript package on NPM: https://www.npmjs.com/package/vitessce. The Python API and Python Jupyter widget are available at https://github.com/vitessce/vitessce-python. The Vitessce Python package is distributed on PyPI at https://pypi.org/project/vitessce. The R API and R htmlwidget are available at https://github.com/vitessce/vitessce-r.

\section{References}

1. Yuan, G.-C. et al. Challenges and emerging directions in single-cell analysis. Genome Biol. 18, 84 (2017).

2. Satija, R., Farrell, J. A., Gennert, D., Schier, A. F. \& Regev, A. Spatial reconstruction of single-cell gene expression data. Nat. Biotechnol. 33, 495-502 (2015).

3. Kharchenko, P. V., Silberstein, L. \& Scadden, D. T. Bayesian approach to single-cell differential expression analysis. Nat. Methods 11, 740-742 (2014). 
4. Wolf, F. A., Angerer, P. \& Theis, F. J. SCANPY: large-scale single-cell gene expression data analysis. Genome Biol. 19, 15 (2018).

5. Zhuang, X. Spatially resolved single-cell genomics and transcriptomics by imaging. Nat. Methods 18, 18-22 (2021).

6. Li, B. et al. Cumulus: a cloud-based data analysis framework for large-scale single-cell and single-nucleus RNA-seq. bioRxiv 823682 (2019).

7. Megill, C. et al. cellxgene: a performant, scalable exploration platform for high dimensional sparse matrices. bioRxiv 2021.04.05.438318 (2021) doi:10.1101/2021.04.05.438318.

8. Samatova, N. F., Breimyer, P., Hendrix, W., Schmidt, M. C. \& Rhyne, T. An outlook into ultra-scale visualization of large-scale biological data. in 2008 Workshop on Ultrascale Visualization 29-39 (2008).

9. Stoeckius, M. et al. Simultaneous epitope and transcriptome measurement in single cells. Nat. Methods 14, 865-868 (2017).

10. Chen, K. H., Boettiger, A. N., Moffitt, J. R., Wang, S. \& Zhuang, X. Spatially resolved, highly multiplexed RNA profiling in single cells. Science 348, aaa6090 (2015).

11. Chen, S., Lake, B. B. \& Zhang, K. High-throughput sequencing of the transcriptome and chromatin accessibility in the same cell. Nat. Biotechnol. 37, 1452-1457 (2019).

12. Miles, A. et al. Zarr. (2020). doi:10.5281/zenodo.4069231.

13. Linkert, M. et al. Metadata matters: access to image data in the real world. J. Cell Biol. 189, 777-782 (2010).

14. Besson, S. et al. Bringing Open Data to Whole Slide Imaging. Digit Pathol (2019) 2019, 3-10 (2019).

15. Moore, J. Next-generation file format (NGFF) specifications for storing bioimaging data in the cloud. (2020). doi:10.5281/zenodo.4282107. 
16. Manz, T. et al. Viv: Multiscale Visualization of High-Resolution Multiplexed Bioimaging Data on the Web. (2020) doi:10.31219/osf.io/wd2gu.

17. Kerpedjiev, P. et al. HiGlass: web-based visual exploration and analysis of genome interaction maps. Genome Biol. 19, 125 (2018).

18. Satyanarayan, A., Moritz, D., Wongsuphasawat, K. \& Heer, J. Vega-Lite: A Grammar of Interactive Graphics. IEEE Trans. Vis. Comput. Graph. 23, 341-350 (2017).

19. Boukhelifa, N. \& Rodgers, P. J. A Model and Software System for Coordinated and Multiple Views in Exploratory Visualization. Inf. Vis. 2, 258-269 (2003).

20. R Core Team. R: A language and environment for statistical computing. (2014).

21. Fang, R. et al. SnapATAC: A Comprehensive Analysis Package for Single Cell ATAC-seq. bioRxiv 615179 (2020).

22. Snyder, M. P. et al. The human body at cellular resolution: the NIH Human Biomolecular Atlas Program. Nature 574, 187-192 (2019).

23. Cutler, Z., Gadhave, K. \& Lex, A. Trrack: A Library for Provenance Tracking in Web-Based Visualizations. (2020) doi:10.31219/osf.io/wnctb.

24. Gratzl, S., Lex, A., Gehlenborg, N., Cosgrove, N. \& Streit, M. From visual exploration to storytelling and back again. Comput. Graph. Forum 35, 491-500 (2016).

25. Petukhov, V. et al. Cell segmentation in imaging-based spatial transcriptomics. Nat. Biotechnol. 1-10 (2021).

26. Codeluppi, S. et al. Spatial organization of the somatosensory cortex revealed by osmFISH. Nat. Methods 15, 932-935 (2018).

27. Preissl, S. et al. Single-nucleus analysis of accessible chromatin in developing mouse forebrain reveals cell-type-specific transcriptional regulation. Nat. Neurosci. 21, 432-439 (2018). 
28. Harris, C. R. et al. Array programming with NumPy. Nature 585, 357-362 (2020).

29. Czech, E., Aksoy, B. A., Aksoy, P. \& Hammerbacher, J. Cytokit: a single-cell analysis toolkit for high dimensional fluorescent microscopy imaging. BMC Bioinformatics 20, 448 (2019).

30. Diehl, A. D. et al. The Cell Ontology 2016: enhanced content, modularization, and ontology interoperability. J. Biomed. Semantics 7, 44 (2016). 


\section{Supplementary Information}

\section{Supplementary Table 1}

\begin{tabular}{|l|l|l|l|l|}
\hline Use case & Number of cells & $\begin{array}{l}\text { Number of } \\
\text { molecules }\end{array}$ & $\begin{array}{l}\text { Number of } \\
\text { genes or } \\
\text { antigens }\end{array}$ & $\begin{array}{l}\text { Image } \\
\text { dimensions at } \\
\text { max zoom }(\mathrm{X} ; \mathrm{Y} ; \\
\mathrm{Z} ; \mathrm{C} ; \mathrm{T})\end{array}$ \\
\hline 1 & 4,085 & 782,300 & 241 & $\begin{array}{l}(5,721 ; 9,392 ; 9 ; \\
3 ; 1)\end{array}$ \\
\hline 2 & 128,119 & NA & 29 & $\begin{array}{l}(12,657 ; 9,488 ; \\
1 ; 29 ; 1)\end{array}$ \\
\hline 3 & NA & NA & NA & $\begin{array}{l}4 \times(1,112 ; \\
1,522 ; 1 ; 61 ; 1)\end{array}$ \\
\hline
\end{tabular}

Size of dataset used in each use case.

\section{Supplementary Table 2}

\begin{tabular}{|l|l|l|}
\hline Name & Description & URL \\
\hline React & $\begin{array}{l}\text { JavaScript API for developing } \\
\text { reactive user interfaces. }\end{array}$ & https://reactjs.org// \\
\hline WebGL & $\begin{array}{l}\text { Low-level JavaScript API for } \\
\text { rendering high-performance } \\
\text { interactive graphics. }\end{array}$ & https://www.khronos.org/web \\
\hline Zarr.js & $\begin{array}{l}\text { A TypeScript implementation } \\
\text { of Zarr. }\end{array}$ & $\underline{\text { https://github.com/gzuidhof/z }}$ \\
\hline Deck.gl & $\begin{array}{l}\text { WebGL-powered framework } \\
\text { for } \\
\text { high-performance } \\
\text { visualization. }\end{array}$ & https://deck.gl/ \\
\hline Viv & $\begin{array}{l}\text { JavaScript library for } \\
\text { multiscale visualization of } \\
\text { high-resolution multiplexed }\end{array}$ & liv \\
\hline
\end{tabular}




\begin{tabular}{|l|l|l|}
\hline & bioimaging data. & \\
\hline HiGlass & $\begin{array}{l}\text { JavaScript library for } \\
\text { visualization of genomic } \\
\text { contact matrices and genome } \\
\text { browser tracks. }\end{array}$ & $\underline{\text { http://higlass.io }}$ \\
\hline Vega-Lite & $\begin{array}{l}\text { JavaScript library } \\
\text { implementing a high-level } \\
\text { grammar of interactive } \\
\text { graphics. }\end{array}$ & $\underline{\underline{\text { el }}}$ \\
\hline
\end{tabular}

Software libraries and web technologies used by Vitessce.

\section{Supplementary Table 3}

\begin{tabular}{|l|l|}
\hline Name & Description \\
\hline Zarr-AnnData & $\begin{array}{l}\text { Annotated data (AnnData) object written to } \\
\text { disk as a Zarr store using the write_zarr } \\
\text { function in the anndata Python package. }\end{array}$ \\
\hline Zarr-Genomic & $\begin{array}{l}\text { Multi-scale genome-mapped quantities } \\
\text { corresponding to per-cluster profiles saved as } \\
\text { a Zarr store. }\end{array}$ \\
\hline Zarr-Raster & $\begin{array}{l}\text { Multi-scale multiplexed image format saved } \\
\text { as a Zarr store. }\end{array}$ \\
\hline OME-TIFF & $\begin{array}{l}\text { Open standard for multi-scale multiplexed } \\
\text { bioimaging data containing OME-XML } \\
\text { metadata and TIFF data. }\end{array}$ \\
\hline JSON & $\begin{array}{l}\text { JSON file adhering to a JSON schema } \\
\text { definition. }\end{array}$ \\
\hline
\end{tabular}

File formats supported by Vitessce. 